\title{
Further simplification of the constraints of four-dimensional gravity
}

\author{
Chopin Sod* \\ Department of Physics, National Cheng Kung University, Tainan, Taiwan
}

\begin{abstract}
The super-Hamiltonian of 4-dimensional gravity as simplified by Ashtekar through the use of gauge potential and densitized triad variables can furthermore be succinctly expressed as a Poisson bracket between the volume element and other fundamental gauge-invariant elements of 3-geometry. This observation naturally suggests a reformulation of non-perturbative quantum gravity wherein the Wheeler-DeWitt Equation is identical to the requirement of the vanishing of the corresponding commutator. Moreover, this reformulation singles out spin network states as the preeminent basis for expansion of all physical states.
\end{abstract}

PACS numbers: 04.60.-m, 04.60.Ds

\section{INTRODUCTION}

Non-perturbative canonical quantization of gravity attempts to overcome the perturbative nonrenormalizability of Einstein's theory by treating the constraints exactly. Ashtekar's seminal simplification of the constraints through the use of gauge connection and densitized triad variables [1] bridged the distinction between geometrodynamics and gauge dynamics by identifying the densitized triad, $\tilde{E}^{i a}$-from which the metric is a derived composite - as the momentum conjugate to an $S O(3, C)$ gauge potential $A_{i a}$. The introduction of spin network states 2] have also yielded discrete spectra for well defined area and volume operators [3]. To the extent that exact states and rigorous results are needed, simplifications of the classical and corresponding quantum constraints are of great importance to the program. These include Ashtekar's original simplification and also Thiemann's observation that $\epsilon_{a b c} \epsilon_{i j k} \tilde{E}^{i a} \tilde{E}^{j b}$ in the super-Hamiltonian constraint is proportional to the Poisson bracket between the connection and the volume operator [4]. Thus it is not unreasonable to expect even more progress from further simplification of the constraints, all the more so if the simplification is naturally associated with spin networks states.

Starting with the fundamental conjugate pair and Poisson bracket,

$$
\left\{\tilde{E}^{i a}(\vec{x}), A_{j b}(\vec{y})\right\}_{P . B .}=-i\left(\frac{8 \pi G}{c^{3}}\right) \delta_{j}^{i} \delta_{b}^{a} \delta^{3}(\vec{x}-\vec{y}),
$$

we shall show that the super-Hamiltonian permits a further remarkable simplification: It is in fact expressible as a Poisson bracket between the volume element and other fundamental invariants, even when the cosmological constant, $\lambda$, is non-vanishing. The leads naturally to an equivalent classical constraint, and to the quantum Wheeler-DeWitt Equation as a vanishing commutator relation. The preeminence of spin network states come naturally from the fact that they are eigenstates of

*Electronic address: cpsoo@mail.ncku.edu.tw the Hermitian volume element operator; and can therefore be used as a basis for all physical states.

\section{GAUGE-INVARIANT ELEMENTS OF 3-GEOMETRY AND THEIR POISSON BRACKETS}

Three physical quantities- the volume element $(\tilde{v})$, the Chern-Simons functional of the gauge potential $(C[A])$, and the integral of the mean extrinsic curvature $(K)$ - form the basic ingredients of 4-dimensional General Relativity as a theory of the conjugate pair of densitized triad and gauge variables, $\left(\tilde{E}^{i a}, A_{i a}\right)$. All are gauge-invariant, but the latter two are in addition also invariant under threedimensional diffeomorphisms i.e. they are elements of 3 -geometry.

Their definitions are as follows:

$$
\tilde{v}(\vec{x}) \equiv \sqrt{\frac{1}{3 !} \epsilon_{a b c} \epsilon_{i j k} \tilde{E}^{i a}(\vec{x}) \tilde{E}^{j b}(\vec{x}) \tilde{E}^{k c}(\vec{x})}=\left|\operatorname{det} E_{a i}\right| .
$$

Its integral over the Cauchy surface, $M$, is the volume, $V=\int_{M} \tilde{v}(\vec{x}) d^{3} x$. The Chern-Simons functional of the Ashtekar connection is

$$
C \equiv \frac{1}{2} \int_{M}\left(A^{a} \wedge d A_{a}+\frac{1}{3} \epsilon^{a b c} A_{a} \wedge A_{b} \wedge A_{c}\right) .
$$

Its characteristic feature is that it satisfies $\frac{\delta C[A]}{\delta A_{i a}}=\tilde{B}^{i a}$ if $\partial M=0$; wherein $\tilde{B}^{i a}$ is the non-Abelian $S O(3)$ magnetic field of $A_{i a}[8]$. The integral of the trace of the extrinsic curvature is

$$
K \equiv \frac{i}{2} \int_{M} E^{a} \wedge\left(D_{A} E\right)_{a}=\int_{M}\left(\tilde{E}^{i a} k_{i a}\right) d^{3} x,
$$

with the observation that the complex Ashtekar connection is $A_{i a} \equiv-i k_{i a}+\Gamma_{i a}$, and $\left(D_{A} E\right)_{a}=d E_{a}+\epsilon_{a}{ }^{b c} A_{b} \wedge$ $E_{c}$, and $\Gamma_{a}$ is the torsionless connection $\left(d E_{a}+\epsilon_{a b}{ }^{c} \Gamma_{b} \wedge\right.$ $\left.E_{c}=0\right)$ connection compatible with the dreibein 1-form $E_{a}=E_{a i} d x^{i}$ on $M$.

With the above definitions and the fundamental relation of Eq.(11), it follows that the following Poisson brack- 
ets hold:

$$
\begin{aligned}
\{\tilde{v}, K\}_{P . B .}= & 3\left(\frac{4 \pi G}{c^{3}}\right) \tilde{v} \\
\{\tilde{v}, C\}_{P . B .}= & \left(\frac{2 \pi G}{i c^{3} \tilde{v}}\right) \epsilon_{a b c} \epsilon_{i j k} \tilde{E}^{i a} \tilde{E}^{j b} \tilde{B}^{k c} \\
\{K, C\}_{P . B .}= & \left(\frac{8 \pi G}{c^{3}}\right) \int_{M}\left(\tilde{B}^{i a} k_{i a}\right) d^{3} x \\
\tilde{H} \equiv\left\{\tilde{v}, i C+\frac{\lambda}{3} K\right\}_{P . B .}= & \left(\frac{2 \pi G}{c^{3} \tilde{v}}\right)\left[\epsilon_{a b c} \epsilon_{i j k} \tilde{E}^{i a} \tilde{E}^{j b}\right. \\
& \left.\left(\tilde{B}^{k c}+\frac{\lambda}{3} \tilde{E}^{k c}\right)\right] .
\end{aligned}
$$

\section{FURTHER SIMPLIFICATION OF THE SUPER-HAMILTONIAN CONSTRAINT}

Expressed in Ashtekar variables, the superHamiltonian constraint for the theory of General Relativity is precisely [1]

$$
\tilde{\tilde{H}}_{0}=\frac{c^{3}}{16 \pi G}\left[\epsilon_{a b c} \epsilon_{\sim} \tilde{\sim}^{i j k} \tilde{E}^{i a} \tilde{E}^{j b}\left(\tilde{B}^{k c}+\frac{\lambda}{3} \tilde{E}^{k c}\right)\right] \approx 0 .
$$

It follows that at the classical level, we may equivalently replace the super-Hamiltonian constraint with the vanishing of a Poisson bracket i.e.

$$
\left\{\tilde{v}, \frac{\lambda}{3} K+i C\right\}_{P . B .}=0 .
$$

The new super-Hamiltonian, $\tilde{H}$, is now a tensor density of weight 1 . Using $\tilde{H} \propto \tilde{\tilde{H}}_{0} / \tilde{v}$ it can be demonstrated that the new super-Hamiltonian constraint together with Ashtekar's transcriptions of the Gauss' law and supermomentum constraints remain a set of first class constraints at the classical level.

Even though we may invoke Poisson bracket-quantum commutator correspondence $\{,\}_{P . B .} \mapsto(i \hbar)^{-1}[$,$] , there$ is no unique prescription for defining a quantum theory from its classical correspondence. The previous observations naturally suggest defining four-dimensional non-perturbative Quantum General Relativity as a theory of the conjugate pair $\left(\tilde{E}^{i a}, A_{i a}\right)$ with super-Hamiltonian constraint imposed as the vanishing commutation relation, $\left[\hat{\tilde{v}}(\vec{x}), \frac{\lambda}{3} \hat{\boldsymbol{K}}+i \hat{C}\right]=0$, together with the requirement of invariance under threedimensional diffeomorphisms and internal gauge transformations.

\section{REFORMULATION OF THE WHEELER-DEWITT EQUATION, AND FURTHER COMMENTS}

Physical quantum states $|\Psi\rangle$ are required to be annihilated by the constraint :

$$
\left[\hat{\tilde{v}}(\vec{x}), \frac{\lambda}{3} \hat{K}+i \hat{C}\right]|\Psi\rangle=0
$$

It is very noteworthy that the Wheeler-DeWitt Equation above is not merely symbolic, but is in fact expressed explicitly in terms of gauge-invariant 3-geometry elements $C$ and $K$.

The formulation is so far not confined to a particular representation or realization of the theory. However it is most interesting that explicit realizations and representations of eigenstates of the volume element operator exist, and they are precisely associated with spin network states! On a spin network, it is known that $\tilde{v}^{2}$ acts in a well-defined manner[3]. Its eigenstates are linear combinations of spin network states of the same vertex valency(number of links) at $\vec{x}$, such that

$$
[\hat{\tilde{v}}(\vec{x})]^{2}\left|\Psi_{v}\right\rangle=v^{2}\left|\Psi_{v}\right\rangle,
$$

has spectrum given by $v=0$ if valency of the vertex at $\vec{x}$ is less than 4 , and $v$ can be computed and may be non-trivial if the valency of the vertex at $\vec{x}$ is equal to or greater than four [3]. In the connectionrepresentation of spin network states, the wave function, $\langle A \mid \Psi\rangle=\langle A| \boldsymbol{\Gamma},\{$ vertices $\},\{j\}\rangle$, is such that for the spin network, $\boldsymbol{\Gamma}$, each link between two vertices at $\vec{x}$ and $\vec{y}$ is associated with a non-integrable phase factor $P\left[\exp \left(i \int_{\vec{x} p}^{\vec{y}} A\right)\right]$ with $A$ in the spin- $j$ representation of the Lie algebra. With appropriate assignments of combinations of Wigner symbols at the vertices, spin network states are gauge-invariant.

Simultaneous eigenstates, $\left|\Psi_{v, \gamma}\right\rangle$, with eigenvalues $(v, \gamma)$ of the volume element operator and the (dimensionless) operator $\hat{\Upsilon} \equiv \frac{\lambda}{3} \hat{K}+i \hat{C}$ are solutions. But since $\hat{\Upsilon}$ does not commute off-shell with the volume element, well defined non-trivial simultaneous eigenstates may not exist.

The operator $\hat{K}$ has in fact been studied by Borrisov, De Pietri and Rovelli[ [5], and its action on loop or nonintegrable phase factor elements of spin network states can be made well-defined. This indicates the action of the operator $\hat{\Upsilon} \equiv \frac{\lambda}{3} \hat{K}+i C$ can also be defined on spin network states. The physical meaning of $\hat{K}$ is not readily apparent in the spin network formulation, but its connection to "intrinsic time" in quantum gravity may nevertheless be deduced from a different perspective. Without resorting to particular representations, it is readily verified that, apart from a multiplicative constant, $K$ is in fact conjugate to the intrinsic time variable i.e. $\{\ln \tilde{v}, K\}_{P . B}=\frac{12 \pi G}{c^{3}}$. In the quantum context $\hat{K}$ is thus proportional to the generator of translations of $\ln \tilde{v}=\ln \left|\operatorname{det} E_{a i}\right|$ which is furthermore a monotonic function of the superspace "intrinsic time variable" $\left(\propto \sqrt{\left|\operatorname{det} E_{a i}\right|}\right)$ discovered by DeWitt in his seminal study of canonical quantum gravity [6] . The operator $K$ is thus a Schwinger-Tomonaga "time-evolution operator" (complete with $i$ ) for an intrinsic time variable.

A few observations on the physical requirements of the inner product and the properties of the operators with respect to Hermitian conjugation are in order. At the classical level $\tilde{v}$ and $K$ are real(according to Eqs. (2) 
and (4)), and it is reasonable to require that the inner product must lead to Hermitian $\hat{\tilde{v}}$ and $\hat{K}$. The Ashtekar connection on $M$ is furthermore understood to be the pullback of the self-dual projection of the spin connection to the Cauchy surface. Thus it is also reasonable to conclude that $A^{\dagger}$ corresponds to the anti-self-dual projection, or the orientation-reversed transform, of $A$. In fact these observations suggest that $C^{\dagger}$ is the Chern-Simons functional for the Ashtekar connection of the manifold $\bar{M}$ with the reversed orientation with respect to $M$. Thus the operator $\hat{\Upsilon}$ is not in general $\mathrm{P}$ and $\mathrm{CP}$-invariant. In this respect, spin network states are holomorphic in $A$ and are therefore also not automatically $\mathrm{P}$-invariant. Further discussions on possible P, CP and CPT viola- tions in Ashtekar theory coupled to matter have been discussed elsewhere[7].

The observations presented here highlight many remarkable features in four-dimensional Quantum General Relativity and behoove further consideration and continued research.

\section{Acknowledgments}

This work has been supported by funds from the National Science Council of Taiwan under Grant Nos. NSC93-2112-M-006-011 and NSC94-2112-M-006-006.

ity, gr-qc/0509049.

[4] T. Thiemann, Phys. Lett. B380, 257 (1996).

[5] R. Borissov, R. De Pietri and C. Rovelli, Class. Quantum Grav. 14, 2793 (1997).

[6] Bryce S. DeWitt, Phys. Rev. 160, 1113 (1967).

[7] L. N. Chang and C. Soo, Phys. Rev. D53, 5682 (1996); C. Soo and L. N. Chang, The Weyl theory of fundamental interactions: is CPT violated ?, hep-th/9702171. L. N. Chang and C. Soo, Class. Quantum Grav. 20, 1379 (2003).

[8] If $M$ is with boundary, the imposition of appropriate boundary conditions, or the introduction supplementary boundary terms should be considered. On the other hand, one can treat $\partial M=0$ as a predictive element of the present reformulation. 